\title{
La résistance du milieu humaniste français à la Justification de Jean Petit et à sa diffusion, 1408-
} 1435

Lucie Jollivet

\section{(2) OpenEdition \\ Journals}

Édition électronique

URL : http://journals.openedition.org/questes/5039

DOI : 10.4000/questes.5039

ISSN : 2109-9472

Éditeur

Les Amis de Questes

\section{Édition imprimée}

Date de publication : 13 juillet 2018

ISSN : 2102-7188

Référence électronique

Lucie Jollivet, «La résistance du milieu humaniste français à la Justification de Jean Petit et à sa diffusion, 1408-1435», Questes [En ligne], 39 | 2018, mis en ligne le 30 juillet 2018, consulté le 01 mai 2019. URL : http://journals.openedition.org/questes/5039; DOI : 10.4000/questes.5039 


\section{La résistance du milieu humaniste français à la Justification de Jean Petit et à sa diffusion, 1408-1435}

\section{Lucie JOLLIVET}

Université Rennes 2, Tempora EA 7468

«Il n'est sacrifice tant agreable a Dieu comme le sang d'un tirant et mauvaiz prince ${ }^{1} »$. Le 23 novembre 1407, le duc de Bourgogne Jean sans Peur fait assassiner son neveu, Louis d'Orléans, frère du roi Charles VI. D'abord écrasé par le poids de ce crime odieux, il avoue aux ducs de Berry et de Bourbon avoir ordonné le meurtre «par l'introduction du dyable ${ }^{2} »$. Mais, très vite, il se reprend et charge une équipe de théologiens dirigée par Jean Petit de préparer sa défense.

Le 8 mars 1408, en la grande salle de l'hôtel Saint-Pol, Jean Petit développe publiquement une véritable apologie du crime : Louis d'Orléans était un tyran, cherchant par tous les moyens à accaparer la couronne et à en priver son frère ; plutôt que de condamner Jean sans Peur, le roi devrait le remercier d'avoir ainsi défendu son honneur et sa vie. Aussitôt après la séance solennelle, le duc de Bourgogne satisfait veut répandre la Justification et en commande plusieurs exemplaires, notamment pour ses fils et son épouse ${ }^{3}$.

${ }^{1}$ Boccace, De Casibus, II, 5, «contra filios tyrannorum», traduction de Laurent de Premierfait, Des cas des nobles hommes et femmes, Paris, BnF, fr. 131. Nous remercions chaleureusement Carla Bozzolo de son aide concernant les traductions de Boccace par Laurent de Premierfait.

${ }^{2}$ Alfred Coville, « Le véritable texte de la justification du duc de Bourgogne par Jean Petit, 8 mars 1408 », Bibliothèque de l'École des Chartes, vol. 72, 1911, p. 58.

${ }^{3}$ Ibid., p. 64. 
Cependant, la diffusion de ce texte heurte brutalement une partie de l'opinion contemporaine et, parmi elle, certains membres du cercle humaniste français, composé d'hommes unis par l'amitié, par la défense du pape Benoît XIII ou encore celle du Bien Commun, notion qu'ils empruntent au chancelier de Florence, Coluccio Salutati ${ }^{4}$. En 1408, Jean Gerson, Pierre d'Ailly et Nicolas de Clamanges ${ }^{5}$, entre autres, rejettent en bloc une légitimation de l'homicide qui pervertit la société et la fait sombrer dans le chaos. Cette première génération d'auteurs est rejointe par des hommes nouveaux comme Alain Chartier $^{6}$ au début du $\mathrm{XV}^{\mathrm{e}}$ siècle. La place de Laurent de Premierfait ${ }^{7}$ dans cet ensemble interroge l'historien car certaines de ses idées font le lien entre les différents auteurs, par-delà la géographie et les générations. Dans certaines de leurs œuvres, ils réagissent vivement à la diffusion de la Justification dans le royaume et au-delà. Il s'agit donc ici de mettre en lumière les principaux éléments d'une écriture que l'on peut qualifier non seulement de « résistante » mais également « de combat».

4 À ce propos, voir Nicole Pons, «La présence de Coluccio Salutati dans le recueil épistolaire de Jean de Montreuil », dir. Pierre Blanc, Dario Cecchetti et Daniela Dalla Valle, Franco-Italica, 1, Paris, Honoré Champion, 1992, p. 9-24 et note suivante.

${ }^{5}$ Concernant l'implication de ces auteurs dans les débats humanistes de l'époque, voir Éric Hicks et Ezio Ornato, «Jean de Montreuil et le débat sur le Roman de la Rose », Romania, t. 98, 1977, p. 34-64 et 186-219 ainsi que Emma Caylay, Debate and Dialogue. Alain Chartier and his Cultural Context, Oxford, Oxford University Press, 2006.

${ }^{6}$ Alain Chartier, Le Quadrilogue invectif, éd. Florence Bouchet, Paris, Champion, 2011 ; Philippe Contamine, «Le Quadrilogue invectif d'Alain Chartier (1422) : texte de circonstance ou œuvre littéraire ? », CRMH, n² 24, 2012, p. 37-50.

${ }^{7}$ Laurent de Premierfait, vers 1365-1418. Voir notamment Un traducteur et un humaniste de l'époque de Charles VI : Laurent de Premierfait, dir. Carla Bozzolo, Paris, Publications de la Sorbonne, 2004. 


\section{La diffusion de la Justification entre 1408 et $1435^{8}$}

Après la séance publique de mars 1408, Jean sans Peur commande plusieurs exemplaires de la Justification, afin d'en faire don à ses proches. Les premiers sont des versions luxueuses et enluminées, destinées à ses fils. La production est ensuite continue jusqu'en $1413^{9}$, les premiers obstacles à la diffusion surgissant lorsque Jean Gerson décide d'obtenir la condamnation des thèses de Jean Petit ${ }^{10}$. Ce dernier meurt en 1411 et ne connaîtra pas les nombreux rebondissements de l'affaire, alors que son texte le rendra célèbre au moins jusqu'à la fin du Xv ${ }^{\mathrm{e}}$ siècle.

Le 4 septembre 1413, suite au retour du parti d'Orléans à Paris derrière le jeune Charles, fils de Louis, Gerson prononce un sermon contre Jean Petit : Rex in sempiternum. Dans les jours qui suivent, il présente une cédule de l'Université, suivie par une requête contre les «sept assertions », c'est-à-dire sept affirmations fausses qui auraient été énoncées par le théologien normand ${ }^{11}$. L'année suivante, grâce à son acharnement, il obtient l'examen par la

\footnotetext{
${ }^{8}$ Nous citons d'emblée l'ensemble des travaux consultés pour cet article se rapportant à la question: Alfred Coville, Jean Petit. La question du tyrannicide au commencement du $\mathrm{XV}^{\mathrm{e}}$ siècle, Paris, Picard, 1932 et «Le véritable texte de la justification... », art.cit.; François Foronda, «Une image de la violence d'État française : la mort de Pierre $1^{\text {er }}$ de Castille », dans Violences souveraines au Moyen Âge. Travaux d'une école historique, dir. Bénédicte Sère, François Foronda et Christine Barralis, Paris, PUF, 2015, p. 249-259; Bernard Guenée, Un meurtre, une société. L'assassinat du duc d'Orléans, 25 novembre 1407, Paris, Gallimard, 1992 ; Bertrand Schnerb, Jean sans Peur : le prince meurtrier, Paris, Payot, 2005 ; Simona Slanica, Krieg der Zeichen. Die visuelle Politik Johanns ohne Furcht und der armagnakisch-burgundische Bürgerkrieg, Göttingen, Vandenhoeck \& Ruprecht, 2002 ; Charity Cannon Willard, "The Manuscripts of Jean Petit's Justification : some Burgundian Propaganda Methods of the Early Fifteenth Century », Studi francesi, 13, 1969, p. 271-280. C'est une discussion avec Jenny Stratford lors du colloque «Debuerit habere regnum» à Lisbonne, en octobre 2016, qui nous a lancé sur la piste de la résistance des humanistes à une forme de propagande imagière de Jean sans Peur. Jenny Stratford a comparé l'iconographie manipulée par le clan bourguignon à l'usage que faisait le roi Richard II du trésor royal. Voir Jenny Stratford, Richard II and the English Royal Treasure, Woodbrigde, Boydell Press, 2012.

${ }^{9}$ Alfred Coville, « Le véritable texte... », art. cit., p. 67-68.

${ }^{10}$ Voir Jean Gerson, CEuvres complètes, éd. Palémon Glorieux, Paris, Desclée, 1973, vol. 10 .

${ }^{11}$ Ibid., p. 171-206.
} 
commission du concile de Paris d'au moins onze manuscrits, ce qui prouve la vigueur de la production. Le 23 février 1414, la Justification de Jean Petit est condamnée par le concile, et tous les exemplaires doivent être détruits par le feu. Deux jours après, les exemplaires remis aux membres du concile sont brûlés sur le parvis de Notre-Dame. Un mois plus tard, la même mise en scène se reproduit. Si beaucoup d'exemplaires ont été copiés et diffusés entre 1408 et 1413, bon nombre sont détruits en 1414. Toutefois, la sentence ne s'applique pas sur les terres bourguignonnes, et l'on suppose que les proches de Jean sans Peur ont pu conserver le leur.

Le 31 novembre 1414, le duc fait d'ailleurs parvenir au comte de Savoie une copie du texte par l'intermédiaire de Jean de la Baume ${ }^{12}$. Cette obstination à diffuser la légitimation de l'homicide éclaire la suite de l'affaire : Gerson refuse en effet de se contenter d'une condamnation de Jean Petit et infléchit son travail en direction d'une mise en accusation de celui qu'il considère comme le véritable coupable, le duc de Bourgogne.

Mais ce dernier est un redoutable adversaire et Gerson est désavoué au concile de Constance en janvier 1416. Les rebondissements s'enchaînent alors : en septembre 1416, un arrêt du Parlement de Paris et des lettres royales interdisent d'affirmer et d'enseigner que le tyrannicide est licite sans jugement préalable $^{13}$. Le 22 avril 1418, une seule proposition parmi les sept assertions énoncées par Gerson est condamnée et la décision de janvier 1416 est annulée. C'est une maigre consolation pour Gerson, d'autant que le 29 mai 1418, les partisans de Jean sans Peur entrent dans Paris. Les revirements sont rapides : l'Université fait amende honorable, l'arrêt du Parlement est cassé. La répression contre les «Armagnacs » met ainsi brutalement fin aux discussions, mais pas à la résistance de Gerson et de ses amis, d'autant qu'à partir de 1422 la

\footnotetext{
${ }^{12}$ Alfred Coville, « Le véritable texte... », art. cit., p. 69.

13 Alfred Coville, Jean Petit..., op. cit., chap. 15, «Le concile de Constance », passim ; Bernard Guenée, Un meurtre, une société, op. cit., p. 255.
} 
Justification connaît un regain d'intérêt grâce à sa retranscription dans les Chroniques de Monstrelet. Un nouveau cycle de copies s'enclenche.

C'est le moment choisi par Nicolas de Clamanges pour faire parvenir son traité De lapsu et reparatione justitie au nouveau duc de Bourgogne, Philippe le Bon. Dans cet ouvrage, d'abord destiné au dauphin Louis de Guyenne avant son trépas en 1415, la réflexion de Clamanges sur la justice s'oriente vers l'absolue nécessité de ramener la paix intérieure, alors que la situation du royaume est catastrophique. Après la mort de Louis, Clamanges semble donc attendre l'opportunité de choisir un nouveau dédicataire. Elle se présente finalement dans la confusion suivant l'assassinat de Jean sans Peur à Montereau, sur les ordres du dauphin, et la signature du traité de Troyes. Clamanges pousse subtilement Philippe le Bon, membre incontesté de la lignée des rois de France, à assumer la succession au trône. Cependant, pour mériter le titre de roi, Philippe doit faire preuve de clémence et pardonner à son ennemi, le dauphin Charles, au lieu de faire imprimer de nouveaux exemplaires de la Justification.

Finalement, ce texte, bien que condamné, est recopié puis imprimé à de multiples reprises jusqu'à l'Époque Moderne, comme en attestent les ouvrages conservés dans plusieurs bibliothèques, européennes et américaines ${ }^{14}$. Les manuscrits du $\mathrm{XV}^{\mathrm{e}}$ siècle sont donc des témoins privilégiés de la diffusion de la Justification à cette période. Ils peuvent être classés en différents ensembles.

Tout d'abord, trois manuscrits correspondent sans doute à des versions « de luxe» commandées par Jean sans Peur pour ses proches, car ils sont

\footnotetext{
${ }^{14}$ Les manuscrits du Xv ${ }^{\mathrm{e}}$ siècle identifiés sont les suivants : Paris, BnF, ms. 3728, fol. 141v ; Paris, BnF, fr. 5732 et fr. 5733 ; Paris, BnF, fr. 5060 et fr. 5061, fol. 2-43; Paris, BnF, fr. 5084 ; Paris, BnF, fr. 17513 ; Paris, BnF, fr. 991, fol. 147-149v ; Paris, BnF, fr. 23428, ainsi que des fragments, Paris, BnF, naf. 23010, fol. 38 ; Chantilly, Musée Condé, mss. 878 et 879, fol 1-41; Mariemont, Musée Warocqué, ms. 83; Vienne, Österreichische Nationalbibliothek, Ms. 2657 ; Bruxelles, Bibliothèque royale, ms. 04373-04376, fol. 153191v; Chicago, Newberry Library, ms. 54.5, fol. 121-164v. Nous avons pour ce faire consulté le catalogue archivesetmanuscrits.bnf.fr et sa bibliothèque numérique, gallica.bnf.fr ; la base de données jonas.irht.cnrs.fr, les sites http://bvmm.irht.cnrs.fr et http://www.arlima.net.
} 
enluminés. Il s'agit des manuscrits de Vienne, ms. 2657, de Paris, BnF, fr. 5733 et de Chantilly, ms. $878^{15}$.

L'illustration du premier est de très belle qualité ${ }^{16}$ et s'inscrit dans un encadrement richement décoré de rinceaux : sur un fond de tours et de clochers, se dresse une tente aux couleurs de la maison de France, séparée du fond par de gros rochers et une forêt. Les deux pans relevés de la tente laissent apparaître une fleur de lys surmontée par une couronne d'or, cette dernière malmenée par un loup l'attirant à lui de sa patte griffue. Alors qu'il s'apprête à en saisir un fleuron entre ses dents, un lion en colère le blesse mortellement au poitrail. Endessous, on peut lire les vers suivants : «Par force le leu rompt et tire, a ses dens et gris la couronne, et le lyon par très grande ire, de sa pate gran coup luy donne ».

L'enluminure du BnF, fr. $5733^{17}$ est moins soignée mais le thème et le quatrain explicatif sont les mêmes ${ }^{18}$. Cette version présente des points communs avec la précédente : selon Alfred Coville, l'écriture est du même style, la scène

${ }^{15}$ Voir les figures 1 et 2 en annexe, ainsi que les mss. Chantilly, Musée Condé, Ms. 878, fol. 2 ; BnF, fr. 5733, fol. 2v ; Vienne, Österreichische Nationalbibliothek, Ms. 2657, fol. 1v. Ils ont été décrits par Eberhard König dans deux catalogues d'expositions : "Frankreich feiert das späte Mittelalter», dans Paris 1400. Les arts sous Charles VI, éd. Elisabeth TaburetDelahaye et François Avril, Paris, Fayard/Réunion des musées nationaux, 2004 ; L'art à la cour de Bourgogne. Le mécénat de Philippe le Hardi et de Jean sans Peur, 1364-1419, éd. Stephen Fliegel et Sophie Jugie, Paris, Réunion des musées nationaux, coll. «Arts du Moyen Âge », 2004, p. 88-105.

${ }^{16}$ Voir figure 1.

${ }^{17}$ Voir note précédente et figure 2.

${ }^{18} \mathrm{Au}$ fol. 2r, on peut lire : «Orléans sans per. », au fol. 78v, «Vive Orléans », ainsi qu'une série de diverses annotations au fur et à mesure du texte. Relevons le remplacement de «derainement trespassé » par «traitrement et meschamment tué », fol. 6 et la mention « injustes et deraisonnables », en lieu et place de «justes et véritables », fol. 67. D'après Alfred Coville, la main qui aurait écrit ces mots daterait sans doute du XVI ${ }^{\mathrm{e}}$ siècle, voir « Le véritable texte... », art. cit., p. 77. Ce manuscrit appartenait à cette époque à un personnage nommé Mansy, comme le prouve la note, fol. 1: «c'est à moy S. Mansy », qui prend nettement parti pour Orléans, s'il est également l'auteur des annotations tendancieuses. Le manuscrit se retrouve ensuite dans la bibliothèque des Carmes Déchaux de Clermont, achetée par Baluze pour Colbert, comme le prouve la cote du fol. 3, «Colb. 6312 ». La bibliothèque colbertine est acquise par le roi Louis XV en 1732. 
et la lettrine sont identiques, ce qui accréditerait l'hypothèse d'un exemplaire destiné à la famille de Jean sans Peur, peut-être à l'un de ses enfants ${ }^{19}$.

Enfin, le Chantilly, ms. $878^{20}$ est de très belle facture : la miniature est riche et soignée, tout comme le décor de rinceaux et la lettrine. Elle représente le même thème que les images précédentes, mais la main est visiblement différente. La scène se déroule dans une clairière cernée par la forêt. Devant l'entrée d'une caverne, le loup attire dans sa gueule un fleuron de la couronne et un lion à visage étonnamment humain le blesse mortellement au cou. Le lion paraît courroucé et tire la langue. Faut-il reconnaître dans le grand nez de l'animal celui de Jean sans Peur? Le quatrain est situé avant l'enluminure, au verso du premier folio et il est suivi d'un préambule. L'incipit est différent de celui des deux précédents manuscrits mais semblable à celui du BnF, fr. 5060, sur lequel nous reviendrons plus loin.

Le message véhiculé par ces trois illustrations est clair : le lion, symbole de courage, de noblesse, de force et figure héraldique du duc Jean sans Peur, sauve la couronne de France du grand danger que représente la convoitise du loup, emblème du duc d'Orléans ${ }^{21}$. Dans l'imaginaire médiéval, la figure du loup est traditionnellement attachée à la cupidité, à la sauvagerie et enfin aux forces diaboliques ${ }^{22}$. L'iconographie souligne cette interprétation, notamment grâce aux griffes et aux dents du loup : soif de pouvoir, violence et lèse-majesté sont habilement suggérées et la colère du lion s'en trouve ainsi justifiée.

Les manuscrits que nous allons maintenant évoquer ne comportent pas d'illustrations. Les seuls à ne contenir que le texte de la première Justification

\footnotetext{
${ }^{19}$ Alfred Coville, « Le véritable texte ... », art. cit., p. 78.

${ }^{20}$ Voir https://www.photo.rmn.fr/archive/03-012007-2C6NU04P3Y2X.html (page consultée le 03 janvier 2017).

${ }^{21}$ Alfred Coville, « Le véritable texte... », art. cit., p. 78.

${ }^{22}$ Carla Casagrande et Silvana Vecchio, Histoire des péchés capitaux au Moyen Âge, Paris, Aubier, coll. « Collection historique », 2009, p. 286 et sq.
} 
sont le Mariemont, ms. $83^{23}$ et le BnF, fr. $5732^{24}$. Dans ce dernier, le texte copié est très proche de celui du fr. 5733, avec cette différence que l'emplacement prévu pour l'illustration est resté vide. Dans toutes les autres versions, la défense élaborée par Jean Petit voisine avec d'autres textes, selon une logique qu'il s'agit maintenant de mettre en évidence.

Dans un premier ensemble, la Justification figure parmi des œuvres qui ont soit un lien avec le duché de Bourgogne, soit avec le dossier du tyrannicide. Ainsi, le Chantilly, ms. 879 rassemble la Justification et une chanson déplorant la mort du duc en 1419. Une main a ajouté des notes en marge des folios 47 et 50, pour souligner la signature de traités de paix avec le Dauphin en 1419. L'assassinat du duc de Bourgogne le 10 septembre de la même année n'en apparaît que plus ignominieux. Le BnF, fr. 5060 contient plusieurs textes, dont celui de mars 1408, mais également celui de la seconde défense rédigée par Jean Petit après la réfutation de l'abbé de Cerisy ${ }^{25}$. La réalisation de ce manuscrit prouve que le clan bourguignon jugeait utile de la faire également circuler, sans doute pour renforcer l'argumentaire en faveur du duc. Le Chantilly, ms. 878 aurait pu servir d'original pour la réalisation de cet ouvrage car tous deux ont le même incipit, qui diffère des autres versions.

Le BnF, fr. 3728 se distingue des ouvrages précédents par son préambule. Il contient également différentes pièces essentielles du dossier, tout comme le

${ }^{23}$ Voir la notice de la base Jonas-IRHT/CNRS pour plus de détails (page consultée le 07 juillet 2017).

${ }^{24}$ Le texte de Jean Petit occupe l'intégralité de l'ouvrage, c'est-à-dire cinquante-cinq feuillets, auxquels il faut donc ajouter l'emplacement pour l'enluminure, resté vide. L'ensemble est réalisé sur papier, la notice de la $\mathrm{BnF}$ indique « fin $\mathrm{du} \mathrm{XV}^{\mathrm{e}}$ siècle ». Au bas du feuillet 56r, on peut lire : «collatio facta de verbo ad verbum », ce qui laisserait à penser que le texte copié correspondrait à celui qui fut pris par plusieurs copistes sous la dictée de Jean Petit, juste après la séance de 1408, en vue de la diffusion, voir Alfred Coville, «Le véritable texte... », art. cit., p. 64-65.

${ }^{25}$ Le premier texte de Jean Petit occupe les fol. $1-47 \mathrm{v}$; ensuite, on trouve la réponse de l'abbé de Cerisy, fol. 48-105v, la seconde Justification, répondant à la défense de Louis d'Orléans, fol. 106-226. 
$\mathrm{BnF}$, fr. $17513^{26}$. Mais au moins un des propriétaires du premier fut un ardent défenseur du duc d'Orléans, ainsi que le prouvent les notes marginales ${ }^{27}$. Par contre, le propriétaire du BnF, fr. $5061^{28}$ et le commanditaire du Chicago, ms. 54.5 étaient tous deux bourguignons ${ }^{29}$. Dans le BnF, fr. 23428, la Justification clôt un ouvrage dédié à l'assemblée du clergé de $1406^{30}$. Enfin, quelques manuscrits ne contiennent qu'une partie de la Justification, parmi d'autres textes ${ }^{31}$.

${ }^{26}$ La Justification, suivie de la défense du duc d'Orléans, couvrent l'intégralité des cent
trente-sept feuillets.
${ }^{27}$ BnF, fr. 3728 , « Cy commance le propos de la majoure de maître Jean Petit... ». Il s'agit du manuscrit décrit sous la cote 3726 par Alfred Coville, «Le véritable texte... », art. cit., p. 81. Sur les cent neuf folios, quarante-et-un sont consacrés à la Justification alors que les suivants sont dédiés à la défense du duc d'Orléans par l'abbé de Cerisy, le texte de la paix de Chartres en 1409 et des lettres des enfants d'Orléans envoyées au roi en 1411. En outre, au folio Av, quelques vers sont écrits d'une main différente de celle du $\mathrm{XV}^{\mathrm{e}}$ siècle, d'après Coville, ibid., mais en étroite relation avec le contenu principal du manuscrit, puisqu'il s'agit d'un distique sur l'envie et l'ambition, suivi d'une louange en vers de l'assassinat de Montereau, présenté comme une juste vengeance et enfin de quelques vers contre Jean Petit, « asne docteur » et «fol maistre ». Une fois encore, le contenu de la Justification produit des réactions violentes.

${ }^{28}$ La Justification est ici suivie de deux extraits des registres du greffe civil du Parlement datant de 1416 et 1413, fol. 44-45, puis de la défense de Louis d'Orléans jusqu'au fol. 99. On trouve également des documents en lien avec l'assassinat de Montereau, fol. 102-128. Le tout se conclut sur la «secrète exclamation, instruit que faict le duc de Bourgoigne envers Dieu, par doléance de ces hayneulx », fol. 140-143 Voir le catalogue en ligne de la BnF.

${ }^{29}$ Voir Paul Saenger, A Catalogue of the pre-1500 Western Manuscript Books of the Newberry Library, Chicago, University of Chicago Press, 1989. La Justification couvre les derniers feuillets, $121-164 \mathrm{v}$.

${ }^{30}$ Ce manuscrit a été décrit par Gilbert Ouy, Les manuscrits de l'abbaye de Saint-Victor : catalogue établi sur la base du répertoire de Claude de Grandrue, 1514. Vol. 1, Introduction, concordances et index; vol. 2, Texte, Turnhout, Brepols, coll. "Bibliotheca Victorina", 1999. Les auteurs des autres textes sont des proches de Jean Petit tels Simon de Cramaud et Pierre aux Bœufs, mais aussi des adversaires comme Pierre d'Ailly. Voir http://jonas.irht.cnrs.fr/manuscrit/73006 (page consulté le 7 juillet 2017).

${ }^{31}$ Dans le catalogue de la BnF, il s'agit du fr.084, un exemplaire des Chroniques de Monstrelet, datant, d'après la notice, du XV ${ }^{\mathrm{e}}$ siècle. Au moins une partie de la Justification fut recopiée dans cet ouvrage et la «propagande » bourguignonne en fut aussitôt relancée. Une partie du texte de Jean Petit fut également recopiée dans la Chronique de Pierre Cochon, il s'agit du manuscrit BnF, fr. 5391. Nous remercions chaleureusement Anh Thy Nguyen pour cette précieuse information. Enfin, le fr. 991 contient divers ouvrages sans rapport avec le duc de Bourgogne et la Justification n'occupe que trois feuillets sur les cent cinquante-deux que compte l'ouvrage. Le BnF, fr. 23010 ne possède que l'incipit aux folios 38-39, voir la notice, http://jonas.irht.cnrs.fr/manuscrit/48162 (page consultée le 07 juillet 2017). Enfin, le Bruxelles, ms. 4373-4376 présente la Justification après des textes variés, notamment le 
Nous pouvons donc conclure que des copies intégrales ou partielles de la Justification ont circulé tout au long du $\mathrm{Xv}^{\mathrm{e}}$ siècle $^{32}$. Dans un premier temps, le clan bourguignon cherche à répandre à la fois le plaidoyer de Jean Petit et sa mise en image, comme le prouvent les emplacements prévus pour les enluminures. Ensuite, le texte est privilégié. Le nombre de versions conservées, malgré les autodafés et les condamnations, tend à démontrer que le duc de Bourgogne s'est servi de ce texte comme d'un outil de propagande destiné à légitimer l'assassinat de Louis d'Orléans et à s'assurer du soutien de l'opinion. Les notes relevées sur certains manuscrits ou le choix des textes accompagnant la Justification prouvent que les rédacteurs et ensuite les propriétaires de ces ouvrages en étaient bien conscients. Les membres du cercle humaniste ne pouvaient donc l'ignorer et leur réaction a été immédiate, comme nous allons le constater.

\section{Une résistance active à la propagande bourguignonne}

Dans l'entourage du roi, la résistance à la propagande du duc de Bourgogne se met rapidement en place. Dans les Grandes Heures, par exemple, le duc de Berry se fait représenter aux portes du Paradis, accompagné de son neveu Louis d'Orléans et de son frère Philippe le Hardi, père de Jean sans Peur $^{33}$. Si cette enluminure peut être interprétée comme la revendication par Jean de Berry d'un rôle politique dans la paix de Chartres de 1409, elle est aussi le reflet du refus de la condamnation posthume du duc d'Orléans par la

Respit de la mort de Jean Le Fèvre et l'Epître Othéa de Christine de Pisan. Elle occupe donc la fin de l'ouvrage, fol. 153-191v.

${ }^{32}$ Nous n'avons pas étudié les manuscrits et imprimés datant d'après le $\mathrm{XV}^{\mathrm{e}}$ siècle car notre article porte sur la résistance des membres du cercle humaniste français, dans la $1^{\text {ère }}$ moitié du $\mathrm{XV}^{\mathrm{e}}$ siècle. Mais dans le cadre d'une recherche plus large, cette étude devrait bien sûr être menée.

${ }^{33}$ Voir en annexe, figure 3. 
Justification de Jean Petit : saint Pierre n'ouvrirait pas les portes du Paradis à un $\operatorname{tyran}^{34}$.

Plusieurs membres du cercle humaniste se trouvent justement appartenir à la chancellerie royale, comme Laurent de Premierfait, qui a achevé vers 1400 sa première traduction du De casibus de Boccace et dédicace en 1409 la seconde au duc de Berry. S'il ne s'exprime pas directement à propos de la Justification et de son retentissement dans le royaume, Laurent insiste particulièrement sur les guerres civiles qui ont finalement eu raison de la République romaine ${ }^{35}$. L'apparition de Pompée sur la scène politique puis son affrontement final avec César permettent à Laurent de revenir plus en détails sur ce thème et de dresser un parallèle saisissant avec sa propre époque. Il souligne tout d'abord la jalousie des protagonistes : «la cité de Romme perillee et detruite par les dommageuses dissensions et guerres civiles qui longuement furent contre Marius et Silla, [...] enflammez de envie l'un contre l'autre ${ }^{36} »$.

Plus loin, l'évocation du corps dépecé de Pompée, la tête coupée et le tronc mutilé identifiable seulement par son anneau ${ }^{37}$, rappelle le cadavre de Louis d'Orléans, retrouvé étendu à terre, le crâne fendu et le poing tranché ${ }^{38}$. À Rome, les parents et amis du grand homme vaincu jurent de « faire la vengeance de Pompée ${ }^{39}$, comme Valentine Visconti et ses enfants, puis ses soutiens, derrière le clan armagnac, jurent de venger Louis. Pour l'humaniste, le meurtre

\footnotetext{
34 Coluccio Salutati, chancelier de Florence, a publié son De tyranno en 1400: la polémique qui fait rage en France se fait l'écho de celle qui agita l'Italie quelques années plus tôt.

${ }^{35}$ Laurent de Premierfait, Des cas des nobles hommes et femmes, Paris, BnF, fr. 131, fol. 180v-185, « queles et quantes destruccions de pays, queles et quantes occisions d'ommes, quelz et quants arsins de temples, de palays, de maisons... ».

36 Ibid., fol. 194.

${ }^{37}$ Ibid., fol. 200.

${ }^{38}$ Voir Françoise Autrand, Charles VI, Paris, Fayard, 1986, p. 252-253 et 354-355.

${ }^{39}$ Laurent de Premierfait, Des cas..., op. cit., fol. 202v-203.
} 
d'un homme d'État provoque toujours une sédition qui affecte tout le corps social $^{40}$.

Quelques années plus tard, le conflit n'est toujours pas résolu. La seconde génération d'humanistes s'empare à son tour du thème de la guerre civile. Dans un poème dénonçant les horreurs d'une guerre «intestine», intitulé Ad detestacionem belli gallici et suasionem pacis, Alain Chartier ${ }^{41}$ revient sur l'épisode de la guerre civile entre Sylla et Marius : «Cette rage toute sillanienne s'empare de Rome, devant laquelle Sylla le furieux se pare du titre d'artisan de la paix, après que Rome ait gardé le silence lors du massacre public de Marius $^{42} »$. Ce dernier est donc présenté comme une victime de la folie meurtrière de Sylla. Comment ne pas penser au quatrain accompagnant l'illustration de la Justification ? Le lion, « dans sa grande ire », blesse le loup qui s'attaque traitreusement à la couronne. Mais ce n'est pas le terme « ira » qui a la préférence de Chartier ; il choisit « rabies » et « insania ${ }^{43}$ » qui soulignent la démence et un comportement irrationnel. Alors que Jean Petit louait la légitime colère de Jean sans Peur, Chartier au contraire la rabaisse au niveau des plus viles passions et lui confère une dimension tragique, renforcée par l'évocation du combat mortel entre les frères ennemis Etéocle et Polynice ${ }^{44}$.

Pierre d'Ailly, à l'automne 1419, rappelle à son ami Jean Gerson, les déboires que lui causèrent «[...] les pseudo-théologiens auxquels vous vous êtes heurtés en grand nombre lors du Concile général, parce qu'ils ne se cachaient

40 Voir Bernard Guenée, Un meurtre, une société, op. cit. et Carla Bozzolo, «La conception du pouvoir chez Laurent de Premierfait », dans Un traducteur et un humaniste de l'époque de Charles VI : Laurent de Premierfait, op.cit., p. 53-68.

${ }^{41}$ Dans Pascale Bourgain-Hemeryck, Les æuvres latines d'Alain Chartier. Édition critique, Paris, CNRS, 1977, p. 225-242.

${ }^{42}$ «Hanc sillana rabies Rome consecuta est, cujus titulo se pacis auctorem Silla seviens appelavit, cum trucidatis Marianis cujus in conspectu Roma sileret », ibid., p. 238, § 67.

${ }^{43}$ «[...] parisiaque tellus talem pertulit insaniam, cum diebus abhominandis », ibid., p. 239.

${ }^{44}$ Ibid., p. 229. 
pas dans les ténèbres [...] mais aboyaient le plus souvent en public ${ }^{45} \gg$. Les défenseurs du duc de Bourgogne sont ici comparés à des chiens, grâce au verbe «latrare ». Le cardinal conclut : « la rage du lion s'abattit cruellement sur vous et sur d'autres, provoquant le massacre inhumain de plusieurs courageux défenseurs de la foi, durant la révolte parisienne ${ }^{46}{ }$.

Le lien entre la colère du lion et la folie de l'acte homicide est clairement établi par Gerson, le duc est l'esclave de passions qu'il est incapable de contrôler : «Ô crime, inimaginable, immortelle haine, blessure inguérissable ${ }^{47}$ ! [...] Que s'éloignent la colère féroce, les inimitiés recuites et les plaintes amères. Que la rancour soit rejetée loin de tous ${ }^{48} »$. Le terme «nefas » désigne depuis l'Antiquité un crime très grave et contre nature. Dans Médée de Sénèque, il est associé à la colère, «furor» et « ira », pour souligner la gravité de la transgression. Plus puissante encore, la haine est le facteur déclenchant le passage à l'acte. La colère du « lion » ne peut être que « féroce »: la métaphore ramène le duc dans le champ des pulsions animales et souligne le caractère monstrueux du crime ${ }^{49}$. Les humanistes tentent ainsi de retourner les arguments de la propagande de Jean sans Peur contre lui et frôlent dangereusement la diffamation. Il est vrai que Jean sans Peur est alors mort depuis quelques

${ }^{45}$ Jean Gerson, Euvres complètes, éd. P. Glorieux, Paris, Desclée, 1973, vol. 2, p. 221 : «[...] pseudo theologorum quales multos esse vos in generali concilio experimento probastis, quia non in occulto nec in tenebris susurrando latuerunt sed saepius in publico latraverunt ».

${ }^{46}$ Ibid., «[...] Hinc scandala multa secuta sunt, hinc potissime superba rabies leonis in vos aliosque strenuos tunc fidei defensores crudeliter saeventis, quorum plures in seditione parisiensi sunt inhumaniter trucidati ».

${ }^{47}$ Jean Gerson, « Deploratio super civitatem », éd. Gilbert Ouy, dans Archives d'histoire doctrinale et littéraire du Moyen Âge, Paris, Vrin, 2004, 71, p. 255-286, ici p. 284 : «O inexcogitabile nephas, immortale odium, numquam sanabile vulnus. [...] Nullum crimen majus hoc inventum est, post illam prodiciosam tui, Christe, mortem ».

${ }^{48}$ Ibid., p. 286 : «Vincat, Pater, immensa pietas abissalem maliciam, ut, ubi abondavit delictum, superhabundet et gracia. Longius abeant ire feroces, veteres inimicie et questus amaritudinis. Rancor procul omnis. [...] cor gravatum sceleribus alleva. [...] Transeat quicquid irarum fuit. [...] Redeat et pacis amor, conciliato pacis auctore ».

49 À ce propos, voir Damien Boquet et Piroska Nagy, Sensible Moyen Âge. Une histoire des émotions dans l'Occident médiéval, Paris, Éditions du Seuil, coll. «L'Univers historique », 2015, ainsi que Carla Casagrande et Silvana Vecchio, Histoire des péchés capitaux, op. cit., p. 94-125. 
semaines, à son tour victime d'un homicide avec préméditation. Certains auteurs tentent alors une manœuvre d'apaisement.

C'est ainsi que, autour de $1419-1420^{50}$, dans son traité sur la justice, le De lapsu et reparatione justitie, achevé à la même période que le poème de Chartier et dédicacé à Philippe le Bon, Clamanges affirme :

Il est notoire que le roi se distingue du tyran par la justice, alors que le roi, par l'exercice de la justice préserve les droits et les libertés du peuple, le tyran au contraire use de l'injustice pour l'opprimer. [...] La justice provoque le pardon et la clémence tempère la rigueur de la justice [...]. La patrie dans laquelle il est [...] permis au prince d'extorquer à ses sujets ce qu'il voudra, selon son bon plaisir [...] n'est rien d'autre qu'un repère de brigands ${ }^{51}$.

Les mots de l'auteur sont, dans le contexte, assez remarquables : Jean sans Peur vient d'être assassiné et Clamanges dénonce le conflit «puéril » qui oppose les Armagnacs aux Bourguignons depuis douze ans. Il insiste sur la nécessité de faire preuve de clémence afin que tout rentre dans l'ordre. Il espère visiblement que le nouveau duc de Bourgogne prendra en main la destinée du royaume et l'implore de mettre fin à la guerre civile en pardonnant au meurtrier de son père.

Les membres du cercle partagent tous cette haute idée de la justice dépassant tous les conflits. Laurent de Premierfait, dans son premier prologue des Cas des nobles hommes et femmes, soulignait déjà que :

Aux nobles principalement affert avoir clemence qui met equitté devant vigueur et veult plus encliner a merci que a vengence sanz saillir hors des termes de justice sanz laquele roys ne roys ne roannes ains sont tirans cruelz et tirannes ${ }^{52}$.

${ }^{50}$ Dans De lapsu et reparatione justitiae, éd. Jérôme Drouart, Paris, 1609, p. 1, Nicolas écrit :«[...] ante annos ferme bissenos sedula mecum mente voluens [...] ad Ludovicum ducem Aquitanorum [...]». Le meurtre a eu lieu en 1407 et «bissenos » indique une période de douze ans.

${ }^{51}$ Ibid., p. 24 , p. 3 et p. 7.

${ }^{52}$ Laurent de Premierfait, Des cas ..., op. cit., fol. 1v-5v. 
Sans le binôme Justice et Pitié, ajoute-t-il, « un roy n'est pas roy ains larron » et son royaume est « une larroniere ${ }^{53} »$. Ces mots, assez véhéments, ont pu inspirer Clamanges lorsqu'il rédigeait son traité ${ }^{54}$. Chartier, quant à lui, en appelle au «Bon roi, [...] dont la patience l'emporte sur la démence, la clémence sur la témérité, car [...] la mansuétude et la bonté ont été domestiquées par l'usage ${ }^{55}$ ». Charles doit ainsi pardonner à Jean sans Peur, malgré les nombreux crimes commis. Mais Chartier écrit son poème avant le meurtre de Montereau en septembre 1419 et ce tragique événement relance la guerre civile : l'espoir de paix a été fugace et le légitime héritier du trône est arbitrairement écarté de la succession.

Les auteurs se sont donc attachés à démontrer que Jean sans Peur, à cause de ses péchés et de son refus obstiné de les confesser, ne pouvait en aucun cas être considéré comme le légitime défenseur de la justice et du roi, ainsi que l'affirmait Jean Petit dans sa Justification. Partant, l'assassinat de Louis d'Orléans ne peut être considéré comme un «beau fait» et il faut alors requalifier l'acte en crime de sang.

\section{La mort de Louis d'Orléans : légitime défense ou homicide volontaire?}

La ligne de défense choisie par Jean Petit justifiait l'effusion du sang de Louis d'Orléans en la présentant comme une prévention nécessaire et la décriminalisait en la transformant en «beau fait». Les enluminures et le quatrain explicatif mettaient particulièrement l'accent sur cette dimension, en

${ }^{53}$ Ibid., fol. 267.

${ }^{54}$ Voir Claude Gauvard, «Les humanistes et la justice sous le règne de Charles VI », dans Pratique de la culture écrite en France au Xv $\mathrm{Xv}^{\mathrm{e}}$ siècle, dir. Monique Ornato et Nicole Pons, Actes du colloque international du CNRS, Paris, 16-18 mai 1992, Turnhout, Brepols, 1995, p. $217-244$.

55 Alain Chartier, Ad detestationem, op. cit., p. 239, § 71 : «Igitur, bone rex, quem [...] patientia vince dementiam, temeritatem clementia reprime. Nam [...] mansuetudinis atque humanitatis usu domantur ». 
accusant le loup de mettre à mal la couronne de France grâce à ses dents et à ses griffes. Les membres du cercle humaniste re-criminalisent le forfait commis par le lion, en favorisant le champ lexical du crime ${ }^{56}$ : «crimen», «scelus », «nefas », «facinus» ainsi que leurs dérivés, «sceleratus », «nefandum», «nefastus », ont donc la préférence des auteurs. Apparait ensuite le vocabulaire lié plus précisément au crime de sang, «homicidium/homicida », « interfectio », « occisio », et au sang versé, « effusus ».

Chartier, s'il ne parle jamais d'homicide, insiste fortement sur la souillure sanglante : «cruentus ». Au début de son poème Ad detestationem belli gallici ${ }^{57}$, alors qu'il évoque les causes de la guerre civile, il décrit : «[... nos mains et nos glaives ruisselants du sang de nos frères ${ }^{58} »$. Le thème du sang qui souille car injustement répandu ${ }^{59}$ se trouve au centre du poème et répond à l'apologie du meurtre de Louis d'Orléans véhiculée à la fois par le texte de Jean Petit, les images illustrant ce texte dans les manuscrits et le quatrain qui légende la Justification. Chartier présente comme révoltante l'idée que les honneurs devraient être la juste rétribution du sang répandu.

Clamanges $^{60}$, de son côté, devient plus pressant voire menaçant dans sa dernière œuvre, qui revêt une dimension plus personnelle ${ }^{61}$ :

${ }^{56}$ Analyse réalisée dans notre thèse, «Les humanistes français face aux crises du début du $\mathrm{XV}^{\mathrm{e}}$ siècle », soutenue à l'Université de Rennes 2 en décembre 2013. Voir en particulier II, 2.2a, « Exprimer le crime... ».

57 Alain Chartier, Ad detestationem, op. cit., p. 231-232.

58 Ibid., p. 233 : «excutite ergo, viri, excutite fraterno sanguine manibus madentes gladios...».

59 À ce propos, voir notre article «Le milieu humaniste français et la réhabilitation de la mémoire de Louis d'Orléans », Humanisme et politique en France à la fin du Moyen Âge, dir. Carla Bozzolo, Claude Gauvard et Hélène Millet, Paris, Publications de la Sorbonne, à paraître.

${ }^{60}$ Nicolas de Clamanges, Expositio super quadraginta septem capitula Isaye, Arsenal, ms. 137.

${ }^{61}$ Ibid., fol. 6v : «Quatuor siquidem esse feruntur peccatorum genera que Dominum pro ultione interpellant. Homicidium, videlicet, ut Abel ad Cayn : "ecce sanguis fratris tui clamat ad me terra" Et in Apocalypsis: "Audivi voces occisorum dicencium: "Quare non defendis sanguinem nostrum?"” ». 
Les péchés qui en appellent à la vengeance de Dieu sont au nombre de quatre, c'est-à-dire l'homicide comme celui d'Abel par Caïn : la voix du sang de ton frère s'élève jusqu'à moi. De même, dans l'Apocalypse, j'entendis les voix de ceux qui avaient été immolés demandant: pourquoi ne fais-tu pas justice de notre sang?

On remarquera que l'auteur est bien moins optimiste que dans le traité de 1420. C'est que le temps a passé et ses espoirs ont encore été déçus. Gerson, à la même période, déplore dans un poème que la mémoire bafouée du duc d'Orléans attende toujours sa réhabilitation : «Que dire de Louis, d'illustre mémoire, fils et frère de roi ? Que dire de son sang qui crie de la terre jusqu'à Dieu $^{62}$ ? ». Dans ces deux passages ${ }^{63}$, l'effusion du sang désigne le coupable à la justice $^{64}$.

En 1415, Gerson concluait ainsi son argumentaire contre la licéité du tyrannicide, «nous disons qu'il n'est pas vraiment un homicide, celui qui n'avait pas l'intention de tuer ${ }^{65} »$, utilisant pour la première fois depuis le début de l'affaire le terme «homicida » et introduisant la notion d'homicide volontaire. Clamanges, dans l'Expositio ${ }^{66}$, en affine la définition : il s'agit d'un acte issu de pensées mauvaises, «fomentés dans le cœur» des coupables avant d'être

${ }^{62}$ Jean Gerson, Euvres, éd.cit., vol. 5, p. 212 : « quid filius regis et frater istius, Ludovicus inclyte memorie, [...] quid immo sanguis suus clamat ad Deum de terra...». Ce discours a été prononcé le 5 mai 1416.

${ }^{63}$ Jean Gerson, Deploratio, éd. cit, p. 277 : « effudisti clarum sanguinem »; ibid., p. 279 : « sanguine cruentis, [...] effusi sanguinis in capite viarum, multo sanguine infecte manus...».

${ }^{64}$ À propos du rôle de la cruentation, voir Claude Gauvard, « De grace especial ». Crime, État et société en France à la fin du Moyen Âge, Paris, PUF, 1991, p.179-189.

${ }^{65}$ Jean Gerson, Euvres, éd. cit, vol. 10, p. 212 : «Rursus dicimus quod in casu talis necessitatis occisor esset innocens apud Deum et non esset vere homicidia quia non intendere occidere sed regem suum a morte imminente protegere, sicut judex non recte intendit interficere sed legibus obedire ». Gerson entend prouver qu'il y a bien eu préméditation et que Louis d'Orléans n'avait aucune intention de nuire à son frère. Ainsi, les arguments de Jean Petit seraient caducs.

${ }^{66}$ Nicolas de Clamanges, Expositio, op. cit., fol. $52 \mathrm{v}^{\mathrm{b}}$ : «"Super fructum magnifici cordis regis Assur", id est super opera mala regis assiriorum, quid arbor mala fructus malos facit, opera inquam de radice cordis procedentia, quia de corde, ut ait Salvator, "exeunt cogiationes male, homicidia, adulteria fornicatione" etc, qui "coinquinantur hominem" ». Commentaire d'Isaïe X:12 puis de Mat., XV:19 et XV:20. 
réalisées, ce qui implique la préméditation. La traitrise prouve le «vilain fait», c'est-à-dire l'intention de tuer. Il peut alors y avoir criminalisation de l'intention, aggravant ainsi les circonstances du meurtre, comme le suggère Chartier : «Des lèvres, on offre la paix à son plus proche parent, mais dans son cour, on lui prépare un guet-apens ${ }^{67} »$. La préméditation rend ce dernier d'autant plus abominable qu'il touche un membre de la famille proche. Gerson personnalise ainsi les Tables de la Loi : « Tu ne tueras point, Tu ne prononceras pas le nom de Dieu en vain, Tu ne tueras point traîtreusement ni avec préméditation $^{68} \gg$.

La diffusion de la Justification, ainsi que son illustration dans certains exemplaires de luxe ${ }^{69}$, par une miniature légendée d'un quatrain explicatif, tentaient de décriminaliser l'acte du 23 novembre en légitimant la loi du talion. $\mathrm{Au}$ contraire, les auteurs du milieu humaniste posent les bases d'une justice moderne, qui fait primer en droit non seulement l'esprit de l'acte mais bien l'acte lui-même. L'homicide avec préméditation est un crime très grave, qui doit impérativement être jugé par les lois humaines. Mais pour être pardonné - c'est bien ici le but poursuivi par les auteurs - le crime doit d'abord être avoué. La clémence royale pourrait alors prendre le relais et rétablir la paix sociale grâce aux lettres de rémission ${ }^{70}$. La justification du meurtre par Jean Petit, puis la diffusion de l'apologie du crime accompagnée d'une iconographie diffamatoire à l'encontre du duc d'Orléans, en entravant le cours naturel de la justice, constituent des pierres d'achoppement qui font trébucher la société tout entière dans le péché. Les auteurs sont persuadés que l'échec de la justice royale a provoqué la colère de Dieu, comme le prouverait la suite implacable de maux s'abattant sur le royaume depuis le début du siècle.

\footnotetext{
${ }^{67}$ Alain Chartier, Ad detestationem, éd. cit., p. 238, § 65.

${ }^{68}$ Jean Gerson, Euvres, éd. cit., vol. 10, p. 214.

${ }^{69}$ Les manuscrits Chantilly, ms. 878, Paris, BnF, fr. 5733 et Vienne, ms. 2657.

${ }^{70}$ Voir Claude Gauvard, « De grace especial », op.cit, chap. 20, p. 895-934.
} 
Ainsi, la correspondance des auteurs choisis tout comme certaines de leurs œuvres montrent une forte résistance à la propagande bourguignonne au sein du milieu humaniste français. Cette résistance est immédiate, forte et durable car elle ne s'éteint pas avec la mort des principaux protagonistes, Jean Petit en 1411 et surtout Jean sans Peur en 1419. Au contraire, le violent trépas de ce dernier semble libérer la parole des auteurs. Sous leur plume, la propagande de Jean sans Peur se retourne contre lui : le lion devient le loup assoiffé de pouvoir et Louis est peint en nouvel Abel, tombé sous les coups d'un oncle jaloux.

Mais la résistance du cercle humaniste dépasse le strict cadre moral ou religieux pour s'inscrire dans le champ de la justice et du droit. Ces préoccupations communes, dépassant le lien générationnel, le cadre géographique ou le strict intérêt philologique pour le renouveau de la culture et de la langue, prouvent que l'éloignement et les crises successives n'empêchent pas les membres du cercle d'être réactifs. S'ils ne peuvent tous être qualifiés d'humanistes au sens étroit, la volonté de participer au Bien Commun les unit. Cette notion peut donc être considérée comme le ciment du premier humanisme français, qui décline après le traité d'Arras, en même temps que s'affaiblit, pour un temps, la nécessité de résister. 


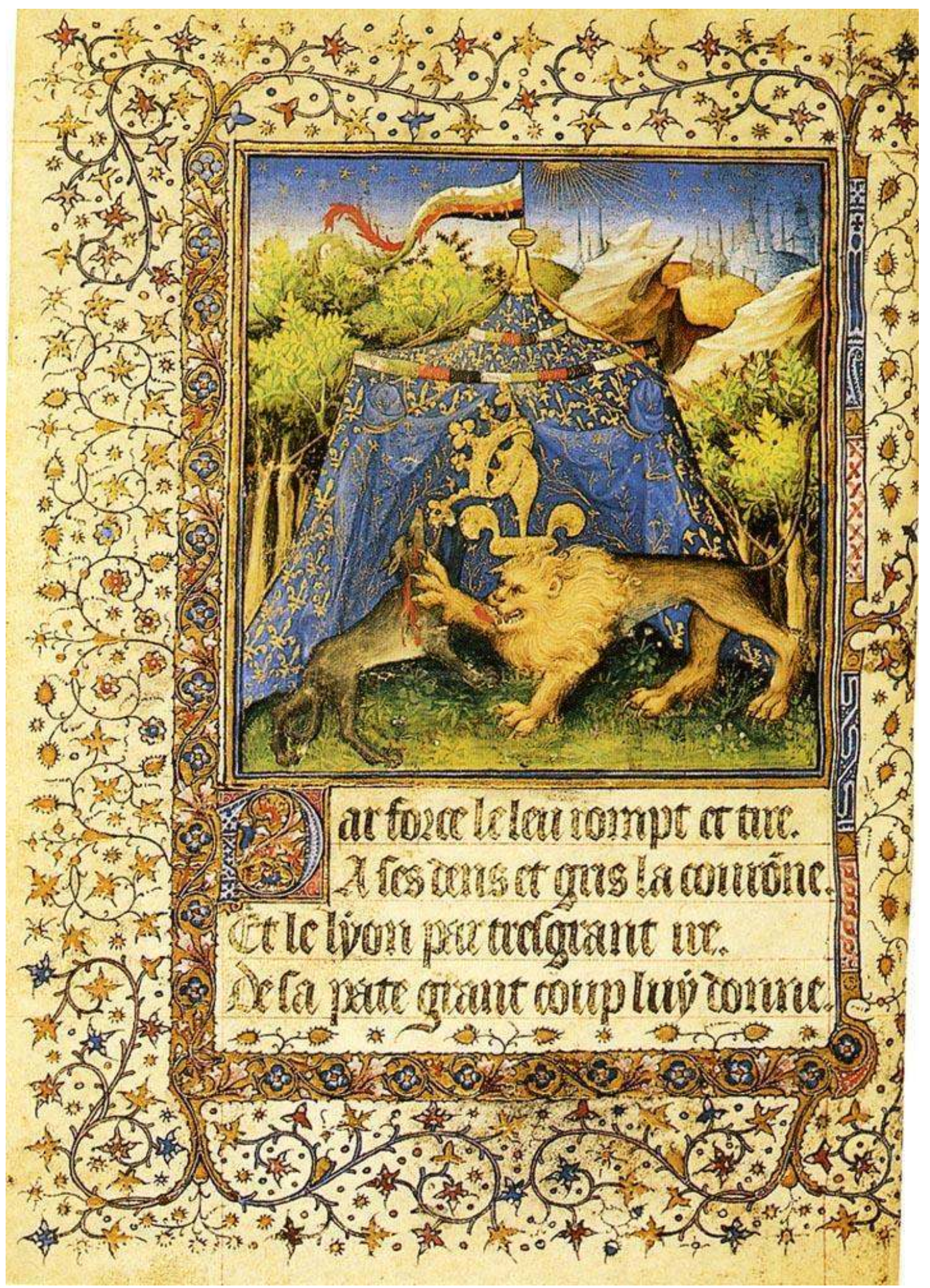

Figure 1 : Vienne, ONB, ms. 2657, fol. 1v.

Source : https://fr.m.wikipedia.org/wiki/Fichier:Loup-Orleans-lion-Bourgogne.jpg Consulté le 10 juin 2017. 


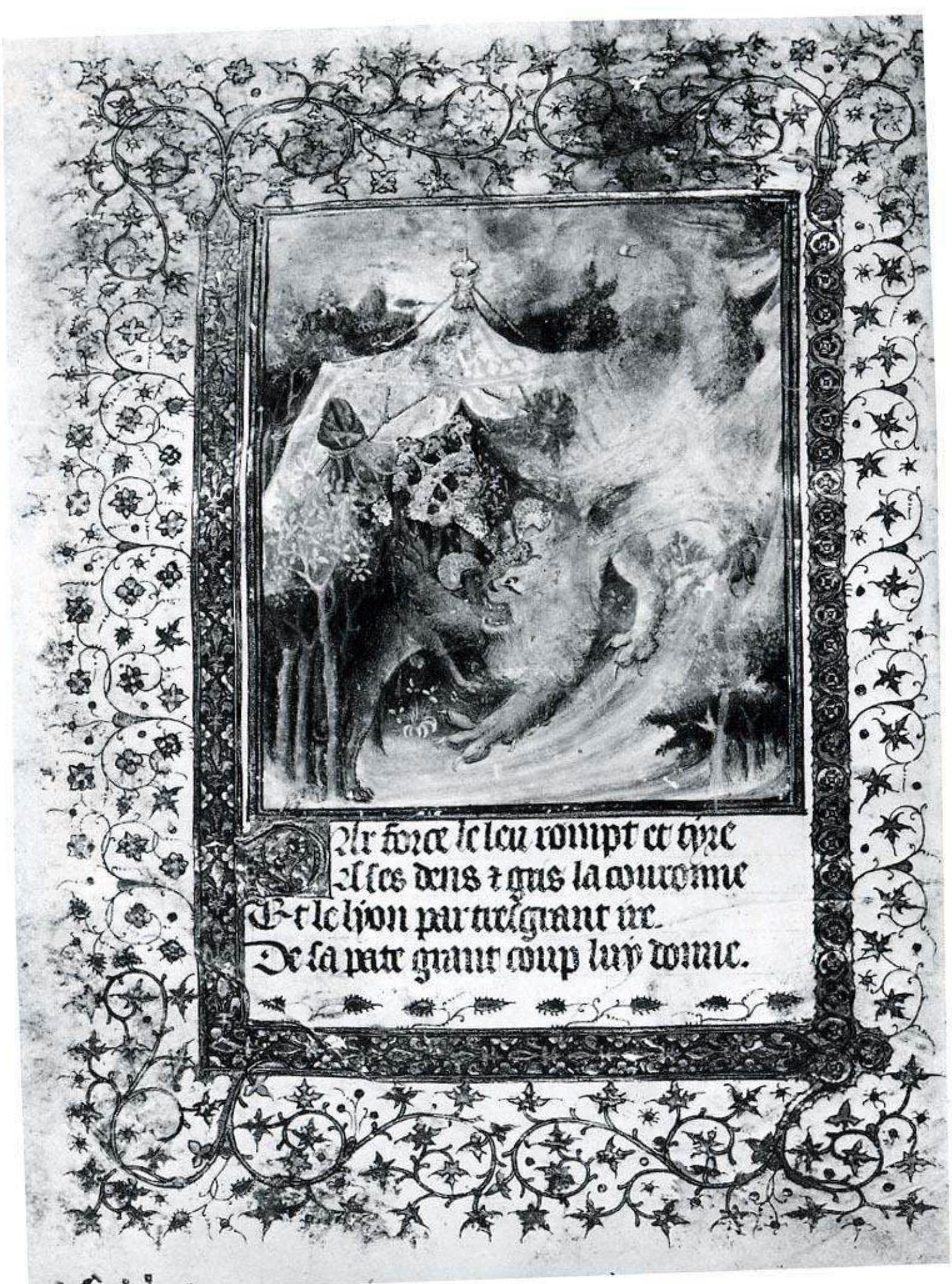

Figure 2 : Paris, BnF, fr. 5733, fol. 2v.

Source : http://employees.oneonta.edu/farberas/arth/Images/ARTH_214images/Manuscripts/1 5_century/douce144group/Paris_BN_fr5733_f1.jpg

Consulté le 10 juin 2017. 


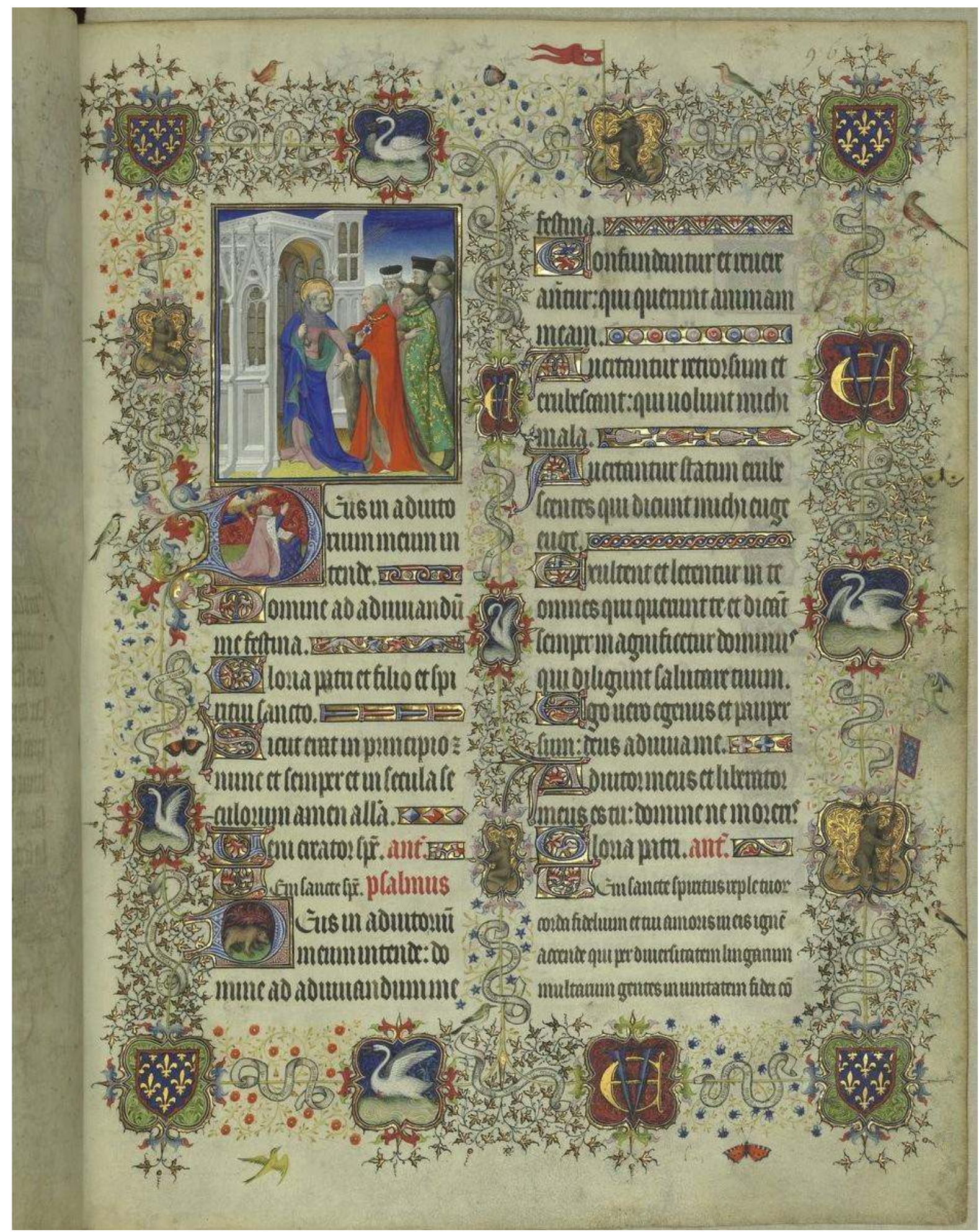

Figure 3 : Grandes heures du duc Jean de Berry, 1409, Paris, BnF, lat. 919, fol. 96.

Source : http://gallica.bnf.fr/ark:/12148/btv1b520004510

Consulté le 10 juin 2017. 